\title{
Madelung Deformity of the Wrist in a 19 Year Old Girl: A Case Report
}

\author{
Elyazid Houass", M. R. Elgaliou, M. Boufetal, R. A. Bassir, M.Kharmaz, M. O. Lamrani, M. S. Berrada
}

Department of Orthopedic Surgery and Trauma, Ibn Sina University Hospital Center Rabat Morocco

Faculty of Medicine and Pharmacy-University Mohamed V Rabat Morocco

DOI: $10.36347 /$ sjams.2021.v09i02.005

| Received: 19.11.2020 | Accepted: 03.12.2020 | Published: 09.02.2021

*Corresponding author: Elyazid Houass

\section{Abstract}

Madelung deformity is a rare congenital anomaly of the wrist caused by asymmetric growth at the distal radius physis secondary to a partial ulnar sided arrest, leading to an ulnar and palmar curvature of the distal radius, positive ulnar variance, and proximal subsidence of the lunate. We report the case of a 19-year-old female with madelung deformity of the right wrist. Conservative management with oral analgesics, activity restriction, and a volar splint was done as the patient was skeletally mature, had only mild pain with no functional limitation or gross deformity.

Keywords: Madelung deformity, wrist, pain, conservative management.

Copyright $(\mathcal{C} 2021$ The Author(s): This is an open-access article distributed under the terms of the Creative Commons Attribution 4.0 International License (CC BY-NC 4.0) which permits unrestricted use, distribution, and reproduction in any medium for non-commercial use provided the original author and source are credited.

\section{INTRODUCTION}

Patients with Madelung deformity most often report gross disfigurement at the wrist caused by the prominence of the relatively long ulna and are often unaware of the deformed radius or the volar subluxation of the hand/carpus, The typical Madelung deformity is associated with a Vickers ligament that creates a tether across the volar-ulnar radial physis that restricts growth across this segment [1].

Treatment depends on the age at presentation, degree of deformity, and magnitude of symptoms and Many patients never require surgical intervention [2].

\section{Report of a CASE}

A 19-year-old female presented to us with pain, deformity, and slight limitation of motion of the wright wrist. The symptoms had started about 6 years back and had gradually progressed since. Pain used to increase with strenuous activity. There was no involvement of any other joint.
Physical examination revealed palmar subluxation of the left hand, dorsal prominence of the ulnar styloid, and ulnar and dorsal bowing of the forearm (Figure-1). Dorsiflexion of the left wrist was slightly restricted.

Anteroposterior and lateral view X-rays of the wrist showed an increased volar bowing of the distal radius, a widened distal radioulnar joint, increased volar tilt of the distal radial articular surface, and an exaggerated ulnar tilt of the distal radius. The carpal bones wedge into the triangular deformity created by the distal radius and a Palmar carpal displacement (Figure-2).

The patient was conservatively managed after discussing the various treatment modalities with her and the parents. She was prescribed oral analgesics, activity restriction, and a volar splint. At the six-month followup, her pain had reduced and she had no complaints. 


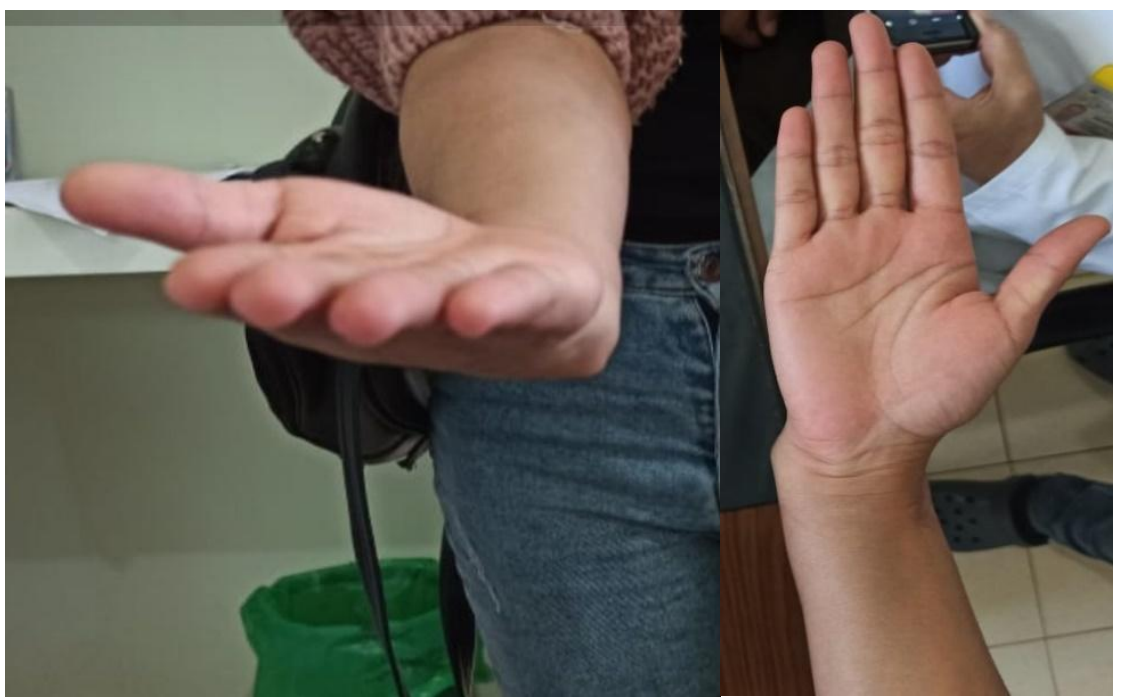

Fig-1: Physical examination revealeing a palmar subluxation of the right hand, dorsal prominence of the ulnar styloid, and ulnar and dorsal bowing of the forearm

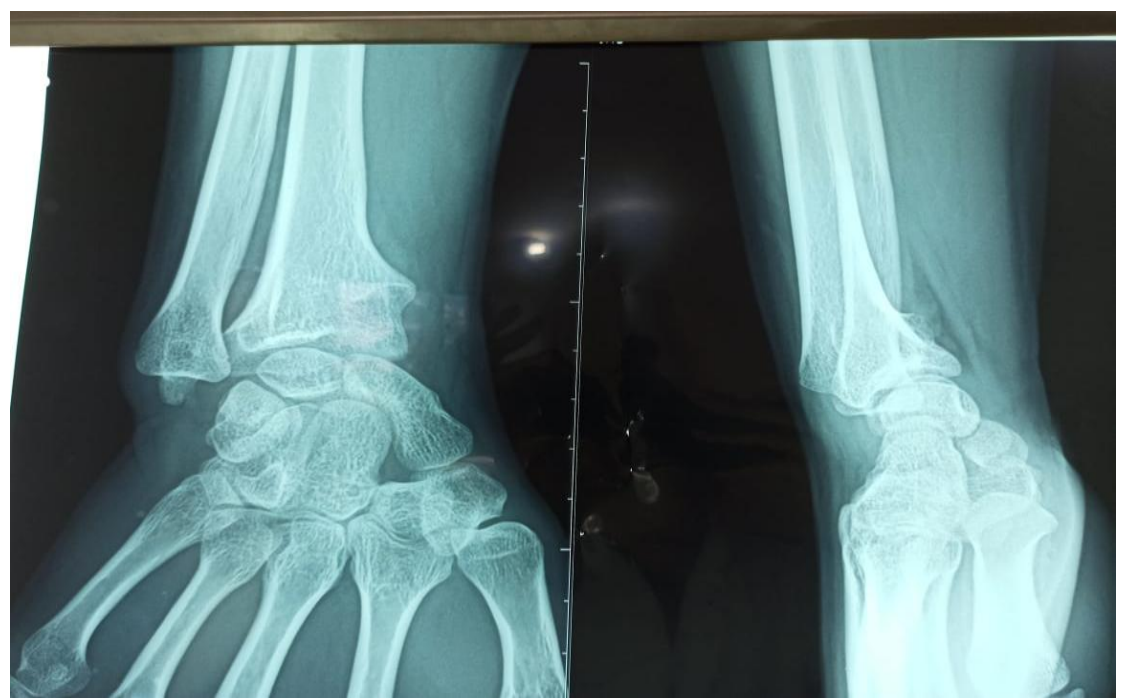

Fig-2: Anteroposterior and lateral view X-rays of the wrist showing an increased volar bowing of the distal radius, increased volar tilt of the distal radial articular surface, and an exaggerated ulnar tilt of the distal radius. The carpal bones wedge into the triangular deformity created by the distal radius and a palmar carpal displacement

\section{DiSCUSSION}

Madelung deformity is described as an idiopathic, progressive curvature of the radius that results in a characteristic anterior subluxation of the hand with respect to the distal radioulnar joint [3, 4]. The disorder was initially described by Madelung in 1878 , before the advent of modern radiography [5].

The disorder is relatively uncommon, occurring in less than $2 \%$ of the general population, and is most prevalent in female patients [6].

Although that the exact etiology of the disease is still unknown, all the evidence suggests that the disorder is related to an abnormal growth of the epiphyseal platein the distal and volar parts of the radioulnar join, Madelung deformity is generally divided into 4 groups according to the etiological factors of the disorder; post traumatic, dysplastic, genetic and idiopathic causes [7].

Individuals who present with the disorder are usually limited in pronation and supination of the involved extremity, although the source of that limitation remains controversial $[3,5]$.

Radiographic findings most often associated with Madelung deformity include increased dorsal and radial bowing of the distal radius, a triangular-shaped carpus, an exaggerated volar and ulnar tilt of the distal articular radial surface, and positive ulnar variance $[8$, 9].

Madelung deformity is usually treated conservatively or surgically depending on the severity of the symptoms 9 . surgery is typically discouraged in 
adolescents and young adults because symptoms tend to subside with age [10].

Surgical treatment is indicated for pain, instability, and restricted wrist movements after a trial of conservative treatment and includes Vickers physiolysis (excision of the Vickers ligament and the abnormal volar-ulnar distal radial physis), radial corrective osteotomy (to correct the position of the articular surface), and distal ulnar shortening osteotomy (in cases with positive ulnar variance) [11].

Conservative management was done in our case as the patient was skeletally mature, had only mild pain with no functional limitation or gross deformity.

\section{Conclusion}

Madelung deformity is a rare congenital anomaly of the wrist caused by asymmetric growth at the distal radial physis secondary to a partial ulnar-sided arrest, Patients generally present in adolescence with pain, deformity, and restricted wrist movements. Treatment can be surgical or non-surgical, conservative management is typically advocated in asymptomatic patients.

\section{REFERENCE}

1. Ghatan AC, Hanel DP. Madelung deformity. JAAOS-Journal of the American Academy of Orthopaedic Surgeons, 2013; 21(6), 372-382.
2. Zebala LP, Manske PR, Goldfarb CA. Madelung's deformity: a spectrum of presentation. The Journal of hand surgery, 2007; 32(9), 1393-1401.

3. Cook PA, Joseph SY, Wiand W, Lubbers L, Coleman CR, Cook AJ, Kean JR. Madelung deformity in skeletally immature patients: morphologic assessment using radiography, CT, and MRI. Journal of computer assisted tomography. 1996 Jul 1;20(4):505-11.

4. Anton JI, Reitz GB, Spiegel MB. Madelung's deformity. Ann Surg. 1938;108:411-436.

5. Vickers D, Nielsen G. Madelung deformity: Surgical prophylaxis (physiolysis) during the late growth period by resection of the dyschondrosteosis lesion. J Hand Surg Br. 1992;17:401-407.

6. Casford B. Madelung's deformity. Available at: http://brighmrad.harvard.edu/Cases/mcr/hcache /205/full.html. Accessed April 19, 2000.

7. Brashear HR, Raney RB. Handbook of Orthopaedic Surgery. 10th ed. St Louis, MO: CV Mosby Co. 1986; 496-497

8. Vender MI, Watson HK. Acquired Madelung-like deformity in a gymnast. J Hand Surg Am. 1988;13:19-21.

9. Lamb D. Madelung deformity. J Hand Surg Br. 1988;13:3-4.

10. Dawe C, Wynne-Davies R, Fulford GE. Clinical variations in dyschondrosteosis: a report on 13 individuals in 8 families. $\mathrm{J}$ Bone Joint Surg Br. 1982;64:377-381

11. Villeco J. Case report and review of the literature: Madelung's deformity. J Hand Ther. 2002, 15:355362. 